\title{
NUMERICAL STUDY OF THERMAL BEHAVIOR OF ANISOTROPIC MEDIUM IN BIDIRECTIONAL CYLINDRICAL GEOMETRY
}

\author{
Rabiâ IDMOUSSA, Nisrine HANCHI, Hamza HAMZA, Jawad LAHJOMRI, Abdelaziz OUBARRA
}

Laboratory of Mechanics, Faculty of Science Aîn Chock, University Hassan II, Casablanca 20100, Morocco

* rabiaa.idmoussa@gmail.com

\begin{abstract}
In this work, we investigate the transient thermal analysis of two-dimensional cylindrical anisotropic medium subjected to a prescribed temperature at the two end sections and to a heat flux over the whole lateral surface. Due to the complexity of analytically solving the anisotropic heat conduction equation, a numerical solution has been developed. It is based on a coordinate transformation that reduces the anisotropic cylinder heat conduction problem to an equivalent isotropic one, without complicating the boundary conditions but with a more complicated geometry. The equation of heat conduction for this virtual medium is solved by the alternating directions method. The inverse transformation makes it possible to determine the thermal behavior of the anisotropic medium as a function of study parameters: diagonal and cross thermal conductivities, heat flux.
\end{abstract}

Keywords: Anisotropic medium, transient heat conduction, coordinates transformation.

\section{Introduction}

Anisotropic materials are present in various industrial applications. Such materials exist either in the natural state as wood and quartz, or industrial, like fibrous materials. Thermal conductivity of these kinds of materials varies according to direction. This makes the heat conduction study delicate due to a cross-derivative terms of the temperature with space variables. When the term cross-derivation is absent, as in the case of orthotropic media, the analysis is simplified, and many studies have been reported in references [1-3].

The case of anisotropic medium has been studied in the context of a permanent [4-8] and a transient conduction regime [9]. The solution in the latter case is limited to the cases of infinite geometries, otherwise there's no analytical solution, hence the need to use a numerical solution, object of our study.

\section{Problem formulation}

The cylindrical anisotropic medium of length $L$ and radius $b$ is illustrated in Fig.1.The left and right sections of are maintained at temperatures $\left(T_{L}, T_{R}\right)$; whereas a radial flux is applied on the surface lateral.

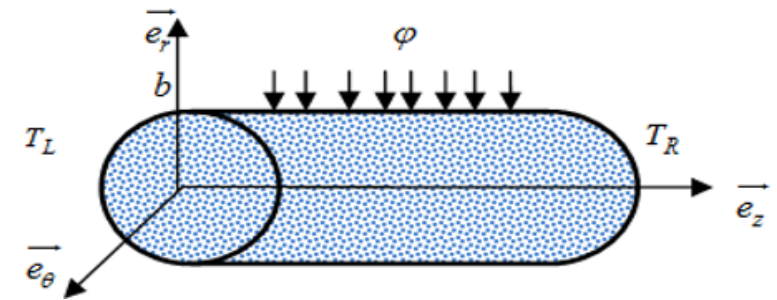

Fig. 1: Three-dimensional representation of the anisotropic cylinder.

Due to the symmetry, the problem is reduced to the twodimensional $(r, z)$ figure, schematized below:

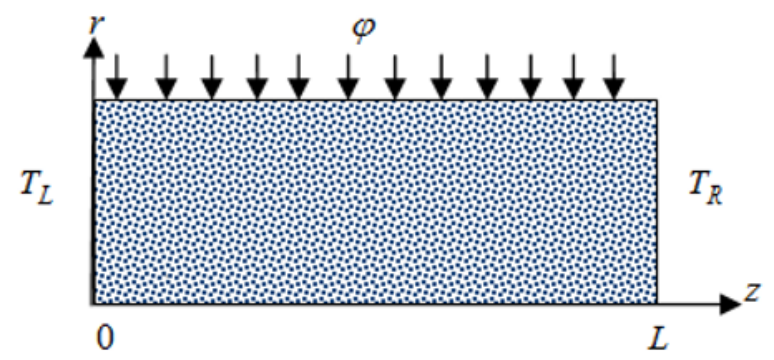

Fig. 2: Bidirectional representation of anisotropic cylinder.

The thermal behavior of the medium studied is governed by:

$k_{r r} \frac{\partial^{2} T}{\partial r^{2}}+\frac{k_{r r}}{r} \frac{\partial T}{\partial r}+k_{z z} \frac{\partial^{2} T}{\partial z^{2}}+2 k_{r z} \frac{\partial^{2} T}{\partial r \partial z}+\frac{k_{r z}}{r} \frac{\partial T}{\partial z}=\rho c \frac{\partial T}{\partial t}$

With $k_{r r}, k_{z z}$ and $k_{r r}$ are respectively, the thermal conductivities according to directions $r, \mathrm{z}$, and $r z$.

The heat fluxes in the directions $r$ and $\mathrm{z}$ according to Fourier's law are given by:

$$
\left\{\begin{array}{l}
\varphi_{r r}=-k_{r r} \frac{\partial T}{\partial r}-k_{r z} \frac{\partial T}{\partial z} \\
\varphi_{z z}=-k_{z z} \frac{\partial T}{\partial z}-k_{r z} \frac{\partial T}{\partial r}
\end{array}\right.
$$

Equation (1) is associated to the boundary conditions:

$T(z=0, r)=T_{L}, \quad T(z=L, r)=T_{R}$

$\left(\frac{\partial T}{\partial r}\right)_{r=0}=0, \quad k_{r r}\left(\frac{\partial T}{\partial r}\right)_{r=b}+k_{r z}\left(\frac{\partial T}{\partial z}\right)_{r=b}=\varphi$

As the resolution of the equation (1) is tricky due to the cross derivative term, we search to bring back in a similar form to isotropic medium, for which the cross term is absent. This is achieved by applying the linear coordinate transformation [6]:

$$
\left(\begin{array}{l}
R \\
Z
\end{array}\right)=\left(\begin{array}{ll}
a & 0 \\
c & 1
\end{array}\right)\left(\begin{array}{l}
r \\
z
\end{array}\right)
$$

with : 


$$
a=\sqrt{\frac{k_{z z}}{k_{r r}}-\left(\frac{k_{r z}}{k_{r r}}\right)^{2}} ; c=-\frac{k_{r z}}{k_{r r}} ; K=k_{z z}-\frac{k_{r z}^{2}}{k_{r r}}=a^{2} . k_{r r}
$$

From this coordinate transformation, Eq. (1) can be written as an isotropic heat conduction equation in the new coordinate system $(R, \mathrm{Z})$ as:

$$
K \frac{\partial^{2} T}{\partial R^{2}}+\frac{K}{R} \frac{\partial T}{\partial R}+K \frac{\partial^{2} T}{\partial Z^{2}}=\rho c_{p} \frac{\partial T}{\partial t}
$$

The heat fluxes in the new coordinates system are given by:

$$
\begin{gathered}
\varphi_{r r}=-a K \cdot r r \frac{\partial T}{\partial R} \\
\varphi_{z z}=-K \frac{\partial T}{\partial Z}-a \cdot K_{r z} \frac{\partial T}{\partial R}
\end{gathered}
$$

These equations are associated with the domain of Fig.3 in the plane $(R, \mathrm{Z})$.

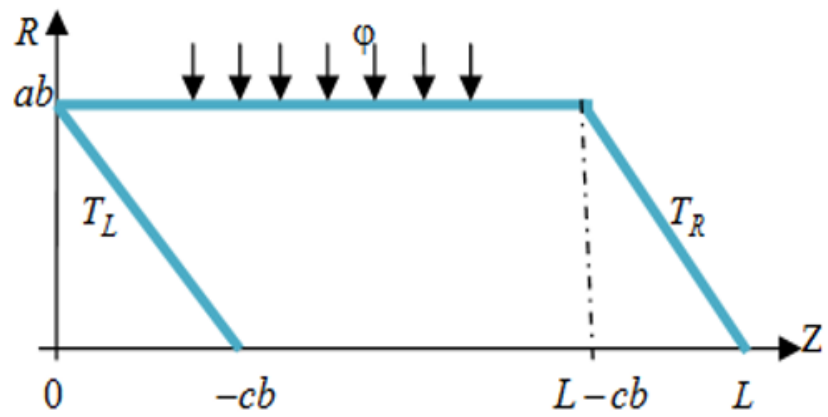

Fig. 3: Computation domain of anisotropic medium in the virtual space.

By introducing the non-dimensional parameters:

$R^{*}=R / b ; Z^{*}=Z / b ; \theta^{*}=\frac{T-T_{R}}{T_{L}-T_{R}} \quad$ and $\quad t^{*}=\frac{t \cdot \alpha_{r r}}{b^{2}}$

with $: \alpha_{r r}=K_{r r} / \rho c_{p}$

The non-dimensional forms of equations (5), (6) and (7) are given as follows:

$$
\begin{gathered}
\frac{\partial^{2} \theta}{\partial R^{* 2}}+\frac{1}{R^{*}} \frac{\partial \theta}{\partial R^{*}}+\frac{\partial^{2} \theta}{\partial Z^{* 2}}=\frac{1}{a^{2}} \frac{\partial \theta}{\partial t^{*}} \\
\varphi_{r r}^{*}=\frac{\varphi_{r r}}{\frac{K_{r r} \cdot\left(T_{L}-T_{R}\right)}{b}}=-a \frac{\partial \theta}{\partial R^{*}} \\
\varphi_{z z}^{*}=\frac{\varphi_{z z}}{\frac{K_{r r} \cdot\left(T_{L}-T_{R}\right)}{b}}=a \cdot c \frac{\partial \theta}{\partial R^{*}}-a^{2} \frac{\partial \theta}{\partial Z^{*}}
\end{gathered}
$$

The associated boundary conditions are given by:

$$
\begin{aligned}
& \theta^{*}{ }_{L}(\mathrm{z}=0, r)=1 ; \theta^{*}{ }_{R}(z=L, r)=0 \\
& \varphi^{*}=\frac{\varphi}{\frac{K_{r r} \cdot\left(T_{L}-T_{R}\right)}{b}} ; a \frac{\partial \theta^{*}}{\partial R^{*}}+c \frac{\partial \theta^{*}}{\partial Z^{*}}=0
\end{aligned}
$$

The determination of the temperature profile in the isotropic dimensional space allows us by the inverse transformation to deduct the evolution of the temperature in the dimensional anisotropic medium.

\section{Numerical solution and Validation}

The numerical method used to solve the above heat conduction equation is the alternating-direction implicit method (ADI). This method is unconditionally stable and gives rise to a tri-diagonal matrix resolved by the THOMAS algorithm [10].

To ensure the validity of numerical analysis, the numerical results were compared with the analytical solution for the case of a steady-state orthotropic medium that is expressed by:

$$
\begin{aligned}
& \theta\left(Z^{*}, R^{*}\right)=1-\frac{Z^{*}}{G}+\frac{4 \varphi^{*} \delta G}{\pi^{2}} \sum_{n=1,2,3}^{\infty} \frac{1}{n^{2}} \frac{I_{0}\left(\lambda_{n} R^{*}\right)}{I_{1} \lambda_{n}} \sin \left(\frac{n \pi R^{*}}{G}\right) \\
& \text { With : } \quad G=L / b, \lambda_{n}=\frac{n \pi}{\delta L / b}, \delta=\sqrt{\frac{K_{r r}}{K_{z z}}}
\end{aligned}
$$

The validation of the numerical code was made by comparing analytical solution with the numerical solution for low value of the cross thermal conductivity $\left(K_{r z} / K_{z z}=0.05\right)$. The comparison concerning an orthotropic medium $\left(K_{r r} / K_{z z}=3\right)$, for a lateral flux $\left(\varphi^{*}=2\right)$ and a form factor $(G=2)$ is reported in Fig.4 and shows a good agreement.

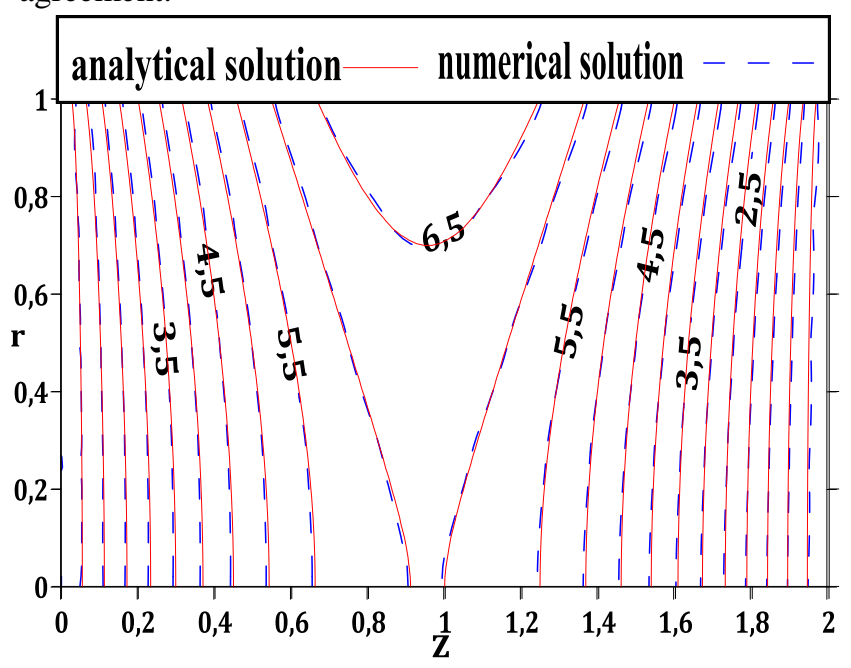

Fig. 4: Validation in the case of an orthotropic medium.

\section{Results and discussion}

The numerical code allows studying transient and steady state thermal behavior of the anisotropic medium according to the study parameters: the ratio of diagonals thermal conductivities, the anisotropic character $\left(K_{r z} / K_{z z}\right)$, the imposed lateral flux $\left(\varphi^{*}\right)$ and the form factor $G$. The results presented in Fig.5 relate to the steady-state thermal behavior of the anisotropic medium for the values of the parameters:

$$
\frac{K_{r r}}{K_{z z}}=0.5,1,2 ; \frac{K_{r z}}{K_{z z}}=0.3,0.6 ; \varphi^{*}=0.5,1 \text { and } \mathrm{G}=2
$$




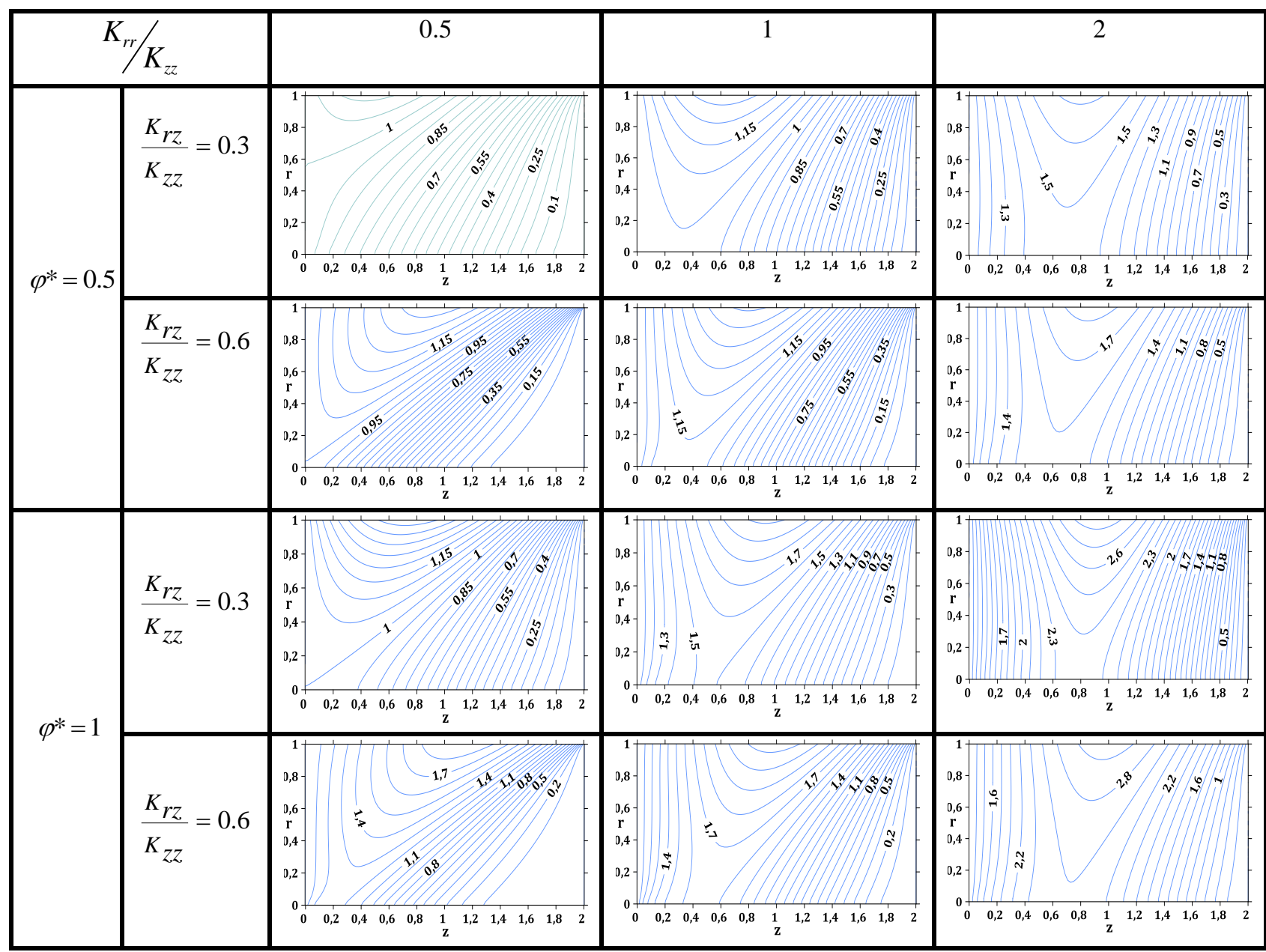

Fig. 5: Steady state temperature profiles in anisotropic cylinder.

From this figure we note that the profile of the isotherms depends on the parameter values: $K_{r r} / K_{z z} ; K_{r z} / K_{z z}$ and $\varphi^{*}$. These parameters have a common effect which results in an increase of the thermal level in the anisotropic medium. The anisotropy factor $\left(K_{r z} / K_{z z}\right)$ modifies in addition the global aspect of the isotherms.

\section{Conclusion}

This study concerns the thermal behavior of an anisotopic medium in two-dimensional cylindrical shape, subjected to a lateral flux and whose sections are at imposed temperatures. This behavior was evaluated by examining the effect of diagonals and cross thermal conductivities in addition to that of the flux. The numerical results show that the anisotropy affects the thermal level as well as the shape of the isotherms.

\section{References}

[1] A.Haji-Sheikh, J.V. Beck, D.Agonafer, Steady-state heat conduction in multi-layer bodies, J. of Heat Mass Transfer 46 (2003), 2363-2379.

[2] H.M. Wang, C.B. Liu, Analytical solution of two-dimensional transient heat conduction in fiber-reinforced cylindrical composites, J. of Thermal Sciences 69 (2013) 43-52.

[3] A. Amiri Delouei, M. H. Kayhani, M. Norouzi, Exact analytical solution of unsteady axi-symmetric conductive heat transfer in cylindrical orthotropic composite laminates, Int. J. of Heat and Mass Transfer 55 (2012) 4427-4436.

[4] N.S.Mera, L.Elliot, D.B.Ingham, An iterative algotithm for singular Cauchy problems for the steady state anisotropic heat conduction equation, J. of Engineering Analysis with Boundary Elements 26 (2002) 157-168.

[5] S. Bouzid, A.C. Boumaaza and M. Afrid, Calcul du champ de température dans un solide anisotrope par la méthode des éléments finis cas bidimensionnel, J.of Energies Renouvelables CISM'08 Oum El Bouaghi (2008) 103 - 111.

[6] C.C. Ma, and S.W. chang, Analytical exact solutions of heat conduction problems for anisotropic multi-layered media, J.of Heat and Mass Transfer, 47 (2004) 1643-1655.

[7] Y.Gu, W.Chen, X.Q.He, Singular boundary method for steady-state heat conduction in three dimensional general anisotropic media, J. of Heat and Mass Transfer 55 (2012) 4837 4848.

[8] R. José, M. Denda, New derivations of the fundamental solution for heat conduction problems in three-dimensional general anisotropic media, J. of Heat and Mass Transfer 54 (2011) 36053612.

[9] M.Ozisik, N. S.M Shouman, Transient heat conduction in an anisotropic medium in cylindrical coordinates, J. of the Franklin Institute 309.6 (1980), 457-472.

[10] N.J. Higham, Accuracy and Stability of Numerical Algorithms, Second Edition. SIAM.p.175, ISBN 978-0-89871802-7 (2002) 